\title{
Tailoring Conjugated Covalent Organic Frameworks in Lieb lattice towards Efficient Photocatalytic Water Splitting
}

\author{
Hongde Yu and Dong Wang* \\ MOE Key Laboratory of Organic OptoElectronics and Molecular Engineering, Department of Chemistry, Tsinghua Univer-
} sity, Beijing 100084, PR China

Supporting Information

\begin{abstract}
Photocatalytic water splitting is a promising solution to sustainable hydrogen production. Recently, conjugated covalent organic frameworks (COFs) with highly designable skeleton and inherent pores have emerged as promising photocatalysts for water splitting. However, structure-property relationships in COFs have not been established and the rational design of COFs with suitable electronic properties and chemical structures for water splitting is challenging. In this work, we proposed a facile strategy that is based on tailoring donor (D) and acceptor (A) building blocks of COFs in Lieb lattice, to achieve visible light absorption, prompt exciton dissociation, and metal-free hydrogen evolution. We constructed eight fully conjugated D-A COFs with a pyrene donor node and different acceptor edges linked by C=C and demonstrated that as compared to the D-D COF, D-A design not only shifted optical absorption to the visible and near-infrared region, but also modified band alignment to trigger overall water splitting. Moreover, the charge-transfer excitation in D-A COFs produced spaceseparated electrons and holes, and the exciton binding energy was substantially reduced due to increased dielectric screening in D-A COFs. Further, we found that the overpotential for hydrogen evolution reaction was suppressed by introducing hydrogen bond interactions in the hydrogen adsorption intermediate to enable metal-free catalysis. Finally, we attained five D-A COFs capable of visible-light-driven hydrogen production in neutral solution without the load of metal co-catalysts. These findings highlight a new route to design COF-photocatalysts and offer tremendous opportunities for regulating COFs towards efficient photocatalytic water splitting and other chemical transformations.
\end{abstract}

\section{INTRODUCTION}

Photocatalytic water splitting is a clean and sustainable energy solution that makes use of inexhaustible sunlight and earth-abundant water to produce hydrogen. ${ }^{1-4}$ Since the discovery of $\mathrm{TiO}_{2}$ photoanode and Pt counter electrode for water oxidation and reduction, ${ }^{5}$ this area has advanced significantly, but it still has a long way to go for practical applications and the search for high-performance photocatalysts continues. ${ }^{6-7}$ Inspired by natural photosynthesis, organic molecular and polymeric materials with tunable band gaps, including g- $\mathrm{C}_{3} \mathrm{~N}_{4},{ }^{8}$ conjugated microporous polymers (CMPs), ${ }^{9}$ linear conjugated polymers, ${ }^{10-12}$ and covalent organic frameworks (COFs), ${ }^{13-20}$ have emerged as metal-free photocatalysts for hydrogen production. COFs are a class of porous materials that integrate organic building blocks into periodic skeleton via reticular chemical reactions. ${ }^{21-22}$ They have received intensive attention as photocatalysts because they combine features such as high crystallinity, tunable pore size and environment of pore walls, large specific surface area, and versatile chemical and topological structures. ${ }^{23-25}$ Recently, two-dimensional (2D) COFs with conjugated structures have been reported to display impressive hydrogen evolution activity, such as azine-linked N3-COF exhibiting a hydrogen evolution rate of $1.7 \mathrm{mmol} \mathrm{g}^{-1} \mathrm{~h}^{-1},{ }^{14}$ and $\mathrm{C}=\mathrm{C}$-linked $\mathrm{sp}^{2} \mathrm{c}$-COFs up to 2.1 mmol g-1 $\mathrm{h}^{-1}$, both in the presence of sacrificial electron donor and Pt co-catalyst. ${ }^{18}$

Despite of the rapid progress in experiment, ${ }^{26-29}$ the structureproperty relationships in photocatalytic COFs have not been established. The rational design of COFs with suitable electronic properties and chemical structures to efficiently split water under visible light is challenging due to the complexity of photocatalytic processes. ${ }^{30}$ A photocatalytic reaction usually involves a series of consecutive photoelectrochemical steps: (1) absorption of photons to generate electron-hole pairs, namely excitons, (2) dissociation of excitons into free charge carriers and charge transport to the catalyst-electrolyte interface, and (3) redox reactions driven by electrons and holes at the interfaces. ${ }^{6,} 31$ Although strategies that combine donor (D) and acceptor (A) building blocks have been used to tune band gaps of COFs, ${ }^{32-33}$ it is not clear how band edge positions of COFs and their alignment with the redox potentials of water will be modified, and they should meet the thermodynamic requirement for water splitting reactions. Moreover, photo-generated electronhole pairs are strongly bounded in organic materials due to the weak Coulombic screening effect, so it is essential to reduce the exciton binding energy and promote charge separation in COFs in order to attain high quantum efficiency. Last but not least, since metal co-catalysts are commonly loaded to accelerate the redox reactions due to lack of active sites on COFs, it is important yet challenging to introduce catalytic sites to COFs and achieve metal-free photocatalysis. $^{31}$

In this work, we proposed a general approach to tailor both chemical and topological structures of COFs towards enhanced absorption in the visible and infrared region, reduced exciton binding energy, and suppressed overpotential for the hydrogen evolution reaction (HER). The main idea is to use donor (D) and acceptor (A) building blocks as node and edge respectively to construct fully conjugated D-A COFs in Lieb lattice. We demonstrated that by choosing acceptor edges with deep frontier orbitals, D-A COFs with narrow band gaps favorable for visible and near-infrared light absorption were attained and they possessed suitable band edge positions for overall water splitting. We also found that D-A design not only gave rise to space-separated electrons and holes, but also increased the dielectric screening in COFs. Additionally, COFs in Lieb lattice with C2 symmetry are lack of flat bands in contrast to Kagome lattice with C3 symmetry, so the effective mass of electrons, holes, and excitons was reduced. All these factors help to reduce the exciton binding energy and thereby promote charge separation. Finally, we unraveled that active sites on COFs for HER 
with low overpotentials can be generated by stabilizing the hydrogen adsorption intermediate via intramolecular hydrogen bond interactions and five D-A COFs were capable of visible-light-driven hydrogen production at neutral solution without the load of metal co-catalysts. These findings offer immense opportunities for chemists to fabricate COFs with desired photocatalytic activity, and will tremendously boost their applications in overall water splitting, ${ }^{6}$ metal-free catalysis, ${ }^{34}$ and other photocatalytic chemical transformations. $^{35-36}$

\section{RESULTS AND DISCUSSION}

\section{Designing D-A COFs in Lieb lattice for photocatalytic water splitting.}

As mentioned above, in the consecutive photoelectrochemical processes, four physical properties are crucial to the design of highly efficient photocatalysts, and they include band gaps, exciton binding energies, band edge positions, and overpotentials for chemical transformations. First, the overall photon energy collected by photocatalysts is determined by their band gaps and absorption spectra. ${ }^{31}$ To enhance light harvest, a suitable band gap is needed to absorb visible and near-infrared light which accounts for over $80 \%$ of the solar power. ${ }^{37}$ However, the band gaps of most COFs and inorganic photocatalysts are above $2.5 \mathrm{eV}$ so they mostly absorb ultraviolet (UV) light (<400 nm). ${ }^{14}$ One way to reduce the band gap is to use alternating donor and acceptor units to build D-A COFs. Although D-A design has been proved effective in modulating band gaps of conjugated polymers ${ }^{38}$ and COFs for organic photovoltaic applications, ${ }^{33},{ }^{39}$ it is unknown how this method will influence other properties like the exciton dissociation and band edge positions that are of great importance for photocatalytic water splitting, and will be explored in the following.

Next, light absorption by COFs generates excitons, i.e. bound electron-hole pairs that can further dissociate into free charge carriers to motivate follow-up redox reactions, in which the exciton binding energy has to be overcome. Although many researchers have realized the importance of exciton dissociation in organic photocatalysts because of weak dielectric screening and strong Coulombic attraction between electrons and holes, ${ }^{40-41}$ it remains to be resolved how to reduce the exciton binding energy in COFs. In principle, the exciton binding energy relies on the exciton mass, static dielectric constant, and exciton radius, ${ }^{42}$ so flat bands and localized electronic states in COFs should be avoided. We noticed that many 2D COFs with $\mathrm{C}_{3}$ symmetry form Kagome lattice, such as N3-COF mentioned earlier, and they display flat bands near the Fermi level arising from non-bonded states, which are adverse to the in-plane charge transport and will increase the effective mass of excitons as well. ${ }^{43-44}$ Instead, COFs in Lieb lattice with C2 symmetry constructed by four-arm nodes are lack of flat bands near the Fermi level (Figure S1), and the effective mass of charge carriers and excitons is reduced and further modulated by tailoring chemical building blocks of COFs. Besides, D-A design usually leads to space-separated electrons and holes, namely enlarged exciton radius, so we propose to design conjugated D-A COFs in Lieb lattice utilizing donor unit as node and acceptor unit as edge, which will reduce not only band gaps but also exciton binding energies of COFs. As will be manifested below, such D-A design can also increase the dielectric screening with the decrease of band gaps, which will benefit exciton dissociation too.

Followed by exciton dissociation, free electrons and holes will transport to the interfaces and trigger the reduction and oxidation of water, namely, hydrogen evolution reaction (HER) and oxygen evolution reaction (OER). These reactions require that not only band edge positions of photocatalysts match the electric potentials of corresponding half reactions, but also photo-generated electrons and holes gain enough energy to overcome the overpotential and drive the reactions. ${ }^{30}$ Unlike inorganic catalysts with partially filled $\mathrm{d}$ or $\mathrm{f}$ orbitals in transition metals, metal-free organic catalysis of low overpotentials is not easily attainable, and design of active sites on organic photocatalysts has attracted increasing attention, especially for HER. ${ }^{8}$ We will demonstrate below that formation of intramolecular hydrogen bonds in the intermediate $\mathrm{H}^{*}$ of hydrogen adsorption on designed D-A COFs $(*)$ helps to stabilize its structure and reduce the overpotential for HER, so metal-free photocatalytic HER in neutral solution is completely feasible.

\section{Modulating band gaps and band edge positions in COFs for overall water splitting under visible light.}

The total energy gathered from sunlight is regulated by the absorption spectroscopy of COFs, and it could be simply indicated by the band gap. ${ }^{31}$ Generally, broad absorption in the visible region of sunlight requires the band gap of $\sim 2 \mathrm{eV}$, and decreased band gap indicates red-shift of spectroscopy and more sunlight harvest. Further, conduction and valence band edge positions of COFs must meet the requirement posed by the electric potentials of half-reactions. ${ }^{37}$ For example, under standard conditions, the conduction band minimum (CBM) of COFs should be sufficiently higher than the water reduction potential of $-4.44 \mathrm{eV}$ (vs. vacuum) to enable electron transfer to water and HER, while the valence band maximum (VBM) should be sufficiently lower than that of the water oxidation potential of $-5.67 \mathrm{eV}$ (vs. vacuum) to enable transfer of hole to water and OER. Although D-A units have been utilized to decrease the band gap in conjugated polymers and COFs, ${ }^{33}$ their impact on the band edge positions, which are essential for photocatalytic reactions, remains to be explored.

COFs are built with nodes and edges as well as linkages between them. In order to attain suitable band gaps and optimal band positions for visible-light-driven water splitting, we constructed D-A COFs by donor nodes, acceptor edges, and conjugated linkages (Scheme 1a). As illustrated in Figure 1a, D-A COFs possess smaller band gaps than D-D COFs due to the small energy offset between the highest occupied molecular orbital (HOMO) of donor node and the lowest unoccupied molecular orbital (LUMO) of acceptor edge. Further, band gaps in D-A COFs exhibit the staggered feature with VB and $\mathrm{CB}$ arise primarily from donor node and acceptor edge respectively, while those in D-D COFs are straddling where VB and CB are both contributed predominantly by node unit.

Based on the above strategy, eight D-A COFs with pyrene donor as node and different acceptors as edge and $-\mathrm{C}=\mathrm{C}$ - linkages have been designed, and they are denoted as PPy-BT, PPy-BT(F), PPyPT, PPy-PT(F), PPy-TDQ, PPy-TzBI, PPy-Q, PPy-Q(F) as sketched in Scheme 1. Herein, PPy represents the pyrene node and it is short for tetrakis(4-formylphenyl)phenyl; BT, PT, TDQ, TzBI, and $\mathrm{Q}$ represent the edges, which are short for benzothiadiazole, pyridal[2,1,3]thiadiazole, thiadiazoloquinoxaline, pyrrolo[3,4f]benzotriazole-5,7-dione, and quinoxaline; F represents peripheral fluorine substitution. And we take $\mathrm{PPy}-\mathrm{Ph}$ as contrast where $\mathrm{Ph}$ stands for phenyl.

$\mathrm{PPy}-\mathrm{Ph}$, namely $\mathrm{sp}^{2} \mathrm{c}-\mathrm{COF}$, are composed of two donor units: $\mathrm{PPy}$ as node and $\mathrm{Ph}$ as edge, linked by $-\mathrm{C}=\mathrm{C}$ - to form a $\mathrm{D}-\mathrm{D} \mathrm{COF}$ with fully $\pi$-conjugated structure. As reported by Jiang and coworkers, ${ }^{18}$ it exhibited highly active hydrogen production of about $2.1 \mathrm{mmol} \mathrm{h}^{-1} \mathrm{~g}^{-1}$ under visible light irradiation in the presence of triethanolamine (TEOA) sacrificial agent and Pt co-catalyst. As shown in Figure 1c and Figure 2a, PPy-Ph is semiconductor with an indirect band gap of $1.72 \mathrm{eV}$ (calculated by HSE06 method), which is consistent with the experimental result of $1.9 \mathrm{eV} .{ }^{18}$ From 
a molecular perspective, the band gap in $\mathrm{PPy}-\mathrm{Ph}$ shows the straddling feature where the HOMO of PPy is higher than that of Ph and the LUMO of PPy lower than Ph, as illustrated by the energy level alignment in Figure 1b. This straddling gap gives rise to the relatively large band gap in D-D COFs. As for the band edge positions (Figure 1c), energies of VBM and CBM in PPy-Ph relative to the vacuum level are $-5.23 \mathrm{eV}$ and $-3.51 \mathrm{eV}$, indicating its capability of photocatalytic HER at $\mathrm{pH}=7$ whereas prohibited OER. These results are in line with the superior HER activity $\left(2.1 \mathrm{mmol} \mathrm{g}^{-1} \mathrm{~h}^{-1}\right)$ and very small OER rate $\left(0.022 \mathrm{mmol} \mathrm{g}^{-1} \mathrm{~h}^{-1}\right)$ of $\mathrm{sp}^{2} \mathrm{c}-\mathrm{COF}{ }^{18}$

In contrast to the D-D COF, D-A COFs possess smaller band gaps (Figure 1c). Taking PPy-BT as an example, it is semiconductor with an indirect band gap of $1.57 \mathrm{eV}$ (Figure 1c and Figure 2c calculated by HSE06 method), which is smaller than PPy-Ph of $1.72 \mathrm{eV}$. From the perspective of building blocks, the LUMO of BT edge is lower than that of PPy node (Figure 1b), which results in the reduced and staggered band gap in PPy-BT. As for the band edge positions (Figure 1c), the energies of VBM and CBM in PPyBT relative to the vacuum levels are $-5.20 \mathrm{eV}$ and $-3.63 \mathrm{eV}$, indicating that it is capable of photocatalytic $\mathrm{HER}$ at $\mathrm{pH}=7$. Although PPy-BT is inactive of OER in neutral solution, PPy-BT(F) with electron-withdrawing F-substitution meets the energy requirement for OER at $\mathrm{pH}=7$, as indicated by the lower VBM energy of -5.45 $\mathrm{eV}$ in PPy-BT(F) (Figure 1c). These results imply that PPy-BT(F) is capable of overall water splitting under visible light. Since the Fsubstitution with strong electron-withdrawing effect lowered the HOMO energy of BT edge, the VBM of COFs is lowered correspondingly as a result of orbital hybridization between node and edge units. This functional group engineering could be a simple and effective approach not only to control the electronic structure of COFs to meet the thermodynamic prerequisite for photocatalytic overall water splitting, but also to modify the microenvironment of pore surfaces. ${ }^{45}$

In addition to PPy-BT and PPy-BT(F), all other D-A COFs possess smaller band gaps than PPy-Ph (Table 1, Figure 1c and Figures S2-S15). Among them, PPy-TDQ has the narrowest band gap of $1.00 \mathrm{eV}$ ascribed to the low-lying LUMO of TDQ edge and its strong electron-accepting ability. A positive correlation is noticed between LUMO energies of acceptor edges and band gaps of D-A COFs: the lower the LUMO energy is in the stronger acceptor, the narrower the band gap, due to the staggered feature of band gaps in D-A COFs. Moreover, the narrowed band gap will give rise to the red-shift in the absorption spectra. As shown in Figure 1d, $\mathrm{PPy}-\mathrm{Ph}$ exhibits a wide absorption from 300 to $650 \mathrm{~nm}$ covering the visible light region of the solar spectrum and peaks at about $500 \mathrm{~nm}$. These results are in good agreement with the experimental absorption spectrum of PPy-Ph which spans from 360 to $620 \mathrm{~nm}$ with a maximum absorption at $497 \mathrm{~nm} .{ }^{18} \mathrm{In}$ comparison, the absorption spectra of D-A COFs are broadened and extend to over $700 \mathrm{~nm}$ when the acceptor becomes stronger and its LUMO gets deeper (Figure 1d and Figure S16). Among them, PPy-TDQ has the broadest absorption spanning from 300 to $900 \mathrm{~nm}$ and could harvest a wide range of visible and near-infrared light. Further, both VBM and CBM positions of COFs were efficiently modified by choosing acceptor edge units with suitable HOMO and LUMO energies to meet the energy requirement for HER and OER. Meanwhile, F-substitution of edge units could simultaneously lower VBM and CBM positions in COFs. As shown in Figure 1c and Figure S17, all these D-A COFs could trigger HER under standard conditions $(\mathrm{pH}=0)$. Four of them, namely PPy-BT(F), PPy-PT, PPy-TzBI, and PPy-Q(F), could serve as photocatalysts for overall water splitting in neutral solution $(\mathrm{pH}=7)$, while PPy-BT and PPy-Q could only trigger HER, and PPy-TDQ and PPy-PT(F) are designed for OER. For those COFs that can only activate half reactions in photocatalytic water splitting, electron donating or accepting sacrificial agents like TEOA or $\mathrm{AgNO}_{3}$ are needed. ${ }^{18}$ Another solution is to combine these COFs to form a Z-scheme heterostructure where a high energy conversion efficiency has also been observed. ${ }^{46}$

We noticed that the D-A design also increased the distance of electron-hole pair. D-D COFs like PPy-Ph possess a straddling gap where VBM and CBM are both distributed on node units and electron and hole are not spatially separated. As illustrated by the partial density of states (pDOS) in Figure 2a, both CBM and VBM are essentially comprised by $\mathrm{C}-2 \mathrm{p}_{z}$ orbitals, confirming the formation of extended $\pi$-conjugation. Further, the charge density at VBM is mainly localized on the pyrene node, while at the CBM it is delocalized over node and edge (Figure $2 b$ ). While for PPy-BT, the DA COF, its band gap is a staggered one where VBM is dominantly contributed by the PPy node while CBM is mainly localized on the BT edge (Figure 2c and Figure 2d). Such spatial separation of electron-hole pair favors exciton dissociation. Staggered band gap and spatial separation of VBM and CBM were similarly observed in other D-A COFs (Figures S2-S15). What's more, D-A COFs other thanr PPy-PT, PPy-PT(F), and PPy-Q possess indirect band gaps where VBM and CBM appear at different $\mathrm{K}$ points and electron and hole have different momenta. Since the transition from CBM to VBM requires the change of electron wave vectors, an indirect band gap tends to inhibit the exciton annihilation and thus prolong its lifetime.

It should be mentioned that all the designed COFs are constituted by $\mathrm{D}$ and A building blocks and share the same Lieb lattice as PPy$\mathrm{Ph}$. And they could be synthesized in lab via Knoevenagel polycondensation reaction with chemicals commonly used to fabricate organic conjugated polymers for photovoltaic applications (Figure S18). ${ }^{28,35,47}$

\section{Reducing exciton binding energies to promote elec- tron-hole separation.}

After photon absorption, Coulomb-bound electron-hole pairs, namely excitons, are generated and they further dissociate into free charges to motivate the electrochemical reactions at the interfaces. The exciton binding energy is an important physical quantity used to gauge the electrostatic interaction between electrons and holes. It is defined as $E_{\mathrm{b}}=E_{\text {elec }}-E_{\text {opt }}{ }^{48}$ where $E_{\text {elec }}$ represents the quasiparticle band gap corresponding to the lower-limit of inter-band transitions, while $E_{\text {opt }}$ represents the optical band gap estimated from the onset of absorption spectra. ${ }^{42}$ Although the importance of excitonic effect in photocatalysts has been well recognized, it remains to be resolved how to reduce the exciton binding energy and facilitate exciton dissociation via the rational design of COFs. ${ }^{26}$ Here, several criteria were proposed and followed which include: (1) decreasing the effective mass of excitons, (2) increasing the static dielectric constant, and (3) increasing the exciton radius and D-A interface area.

In principle, electronic properties of COFs are determined by both chemical building blocks and topological structures. When node units with $\mathrm{C}_{3}$ symmetry are used to construct COFs in Kagome lattice, flat bands typically appear at the VBM or CBM (Figure S1 and Figure S19). ${ }^{43-44}$ These flat bands are formed by nonbonding orbitals of node and edge units and have very large effective mass of electron or hole, which could lead to intriguing magnetic phenomenon but is not desired for high-performance charge transport. ${ }^{43}$ Since the effective mass of exciton could be defined as $1 / m^{*}=1 / m_{\mathrm{e}}{ }^{*}+1 / m_{\mathrm{h}}{ }^{*},{ }^{42}$ where $m_{\mathrm{e}}{ }^{*}$ and $m_{\mathrm{h}}{ }^{*}$ stand for the effective mass of electron and hole respectively, the flat band at VBM or CBM could also give rise to large exciton mass and binding energy. In contrast, COFs constructed by node units with $\mathrm{C}_{2}$ or $\mathrm{C}_{4}$ symmetry, 
such as PPy, porphyrin, and phthalocyanine, will form Lieb lattice. ${ }^{43}$ In Lieb lattice, both valence and conduction bands are nonflat bands arising from conjugated bonding/antibonding orbitals, which give rise to the small effective mass of electron, hole, and exciton. So, in order to avoid flat bands near the Fermi level and reduce the exciton binding energy, we have chosen PPy node and constructed COFs in Lieb lattice.

The static dielectric constant is used to measure Coulombic screening effect in the solid lattice. With the increase of static dielectric constant, the electrostatic interaction between electron and hole decreases, which will lead to small exciton binding energy. In organic solids, the static dielectric constant is usually between 3-4, which is significantly lower than inorganic crystals such as photovoltaic perovskite materials. ${ }^{49}$ Thus, to promote exciton dissociation, we need to enlarge the static dielectric constant of COFs. In low-frequency or static electric fields, dielectric response comes from two main sources, namely ionic and electronic contributions, which can be written as $\varepsilon_{\mathrm{s}}=\varepsilon_{\mathrm{s} \text {,ion }}+\varepsilon_{\mathrm{s} \text {,elec. }}{ }^{50}$ The ionic response $\left(\varepsilon_{\mathrm{s}}\right.$, ion) arises from the motion of atoms which will generate oscillating dipole as with infrared active lattice vibrations and is of particular importance to inorganic solids. In addition to the ionic contribution, the electronic response ( $\varepsilon_{\mathrm{s} \text {,elec }}$ ) accounts for change of the electronic polarizability with the electric field, and it is the dominating mechanism in organic solids especially nonpolar materials. According to Lorentz oscillator model, the electronic static dielectric $(\varepsilon$ s,elec $)$ can be related to the optical absorption frequency $\left(\omega_{\mathrm{e}}\right)$ by $\varepsilon_{\mathrm{s} \text {, elec }}=1+$ $N \mathrm{e}^{2} /\left(m \varepsilon_{0} \omega_{\mathrm{e}}{ }^{2}\right.$ ), where $m$ and $N$ are the mass and number (per unit volume) of oscillators, respectively and $\omega_{\mathrm{e}}$ represents the resonance frequency of oscillation corresponding to the average electronic excitation energy. ${ }^{50}$ So the static dielectric constant will increase with the narrowing of band gap, bringing more effective screening of electrostatic interactions between electron and hole. Such a trend was observed in designed D-A COFs, as illustrated in Figure 3a and Figure S20. Thereby the exciton binding energy $E_{b}$ was substantially reduced with narrowed band gap in D-A COFs as implied by Figure $3 b$. $\mathrm{PPy}-\mathrm{Ph}\left(\mathrm{sp}^{2} \mathrm{c}-\mathrm{COF}\right)$ is the $\mathrm{D}-\mathrm{D} \mathrm{COF}$ and it has an exciton binding energy of about $1200 \mathrm{meV}$, while all D-A COFs have smaller exciton binding energies and PPy-TDQ exhibits the smallest one of $700 \mathrm{meV}$ (Table 1). Thus, design of D-A COFs in Lieb lattice with reduced optical band gaps has been demonstrated to effectively increase the dielectric screening effect and reduce the exciton binding energy.

A well-designed D-A interface can help avoid the recombination of electrons and holes, and large boundary area profits charge separation. As demonstrated above, D-A COFs have staggered band gaps in which the VBM is mainly localized on donor node while the CBM on acceptor edge. These conjugated donor or acceptor units will further stack in the out-of-plane direction to form a onedimensional channel for hole or electron transport. These divided channels represent one of unique features of 2D conjugated COFs and will enlarge the D-A interface area to boost charge separation and inhibit recombination of electrons and holes (Figure 3c). Meanwhile, in D-A COFs with indirect band gaps, the transition from CBM to VBM is inhibited due to the momentum change of electrons, and thus the quenching of electron-hole pairs through radiative or non-radiative pathways is suppressed, which will enhance the quantum efficiency. ${ }^{50}$ Further, the inherent pores in 2D COFs provide predesigned chemical environment for water splitting reactions so that the migration distance for charge carriers is shortened, while the large surface area also provides adequate space for high-load co-catalysts as well as for engineering active sites to achieve metal-free catalysis (Figure 3d and Figures S21-S22).
Suppressing overpotentials via intramolecular hydrogen bonds towards metal-free hydrogen production.

After photo-generated excitons dissociate into electrons and holes, these free charge carriers at CBM and VBM will be transferred to water and trigger the redox reaction. To enable photocatalytic hydrogen production, CBM in COFs must be sufficiently higher than the reduction potential of water in neutral solution, namely -4.03 $\mathrm{eV}$ (vs. vacuum), so adequate driving force can be exerted to surpass the overpotential arising from the reaction mechanism of HER. However, high CBM often means the wide band gap and may cause the blue-shift in absorption spectroscopy which is not favored for sunlight harvesting. So, we should design active sites on pore walls of COFs to lower the overpotential of reaction and boost the hydrogen evolution rate driven by visible light. In contrast to inorganic metal co-catalysts with partially filled d orbitals, such as Pt and $\mathrm{Co}\left(\mathrm{NO}_{3}\right)_{2}$, organic semiconductors lack of active sites often show high overpotentials for HER. A very recent work showed that Nsite on the linkage of COFs, including imine, azine, and azo groups , could serve as active catalytic sites for metal-free HER. ${ }^{30}$ The overpotential for HER can be estimated from the Gibbs free energy change of hydrogen adsorption on the catalyst $\left(\Delta G\left(\mathrm{H}^{*}\right)\right),{ }^{51}$ so it is essential to reduce $\Delta G\left(\mathrm{H}^{*}\right)$, especially the hydrogen adsorption energy by stabilizing the intermediate $\mathrm{H}^{*}$. However, except for highly reactive organic radicals with unpaired electrons, the adsorption of hydrogen on closed-shell organic semiconductors needs to break a chemical bond and produce unstable radical intermediate, which gives rise to high overpotentials $\Delta G\left(\mathrm{H}^{*}\right)$. Based on the above argument, we proposed to design active sites on weak chemical bonds and introduce non-covalent intramolecular interactions such as hydrogen bonds to stabilize $\mathrm{H}^{*}$ and thereby reduce the overpotential for HER.

We took PPy-BT as an example to illustrate the catalytic activity of designed D-A COFs for hydrogen evolution. As shown above, the photo-generated electron at CBM in all D-A COFs is mainly located at the edge unit. So, we investigated the Gibbs free energy change of HER on all potential sites at the BT edge under standard conditions, as shown in Figure 4a. We noticed that hydrogen adsorption onto the $\mathrm{S}$ site is most unfavored in energy because $\mathrm{S}$ atom tends to form only two $\sigma$ bonds. The $\mathrm{C}$ site on -CN group attached to the $\mathrm{C}=\mathrm{C}$ linkage also showed relatively high overpotential because hydrogen adsorption on it would break the strong $\mathrm{C} \equiv \mathrm{N}$ bond. Whereas the $\mathrm{C}$ site on the BT edge possessed moderate $\Delta G\left(\mathrm{H}^{*}\right)$, since adsorption of $\mathrm{H}$ atom on aromatic $\mathrm{C}$ would break a $\pi$ bond and change the hybridization of $\mathrm{C}$ from $\mathrm{sp}^{2}$ to $\mathrm{sp}^{3}$. The N2 site on the BT edge showed the smallest overpotential of only $0.26 \mathrm{eV}$, which could be attributed to the formation of an intramolecular hydrogen bond with the adjacent $\mathrm{N}$ on -CN group (Figure $4 \mathrm{~b}$ ). This hydrogen bond stabilized the $\mathrm{H}^{*}$ structure, but such interaction is lacking on the N1 site of BT edge. These results manifested that the overpotential for HER could be substantially reduced by introducing intramolecular hydrogen bond interactions, and active sites for HER were available on COFs. By contrast, the absence of N-containing active sites on $\mathrm{PPy}-\mathrm{Ph}\left(\mathrm{sp}^{2} \mathrm{c}-\mathrm{COF}\right)$ raised the overpotential to $0.72 \mathrm{eV}$ and inhibited metal-free HER with low-energy excitons (Figure S23). Further, we would like to point out that the -CN group participating in the hydrogen bond formation can be introduced to COFs during the formation of $-\mathrm{C}=\mathrm{C}$ - linkage via Knoevenagel polycondensation reaction, ${ }^{18,52-53}$ and it exists in other conjugated DA COFs designed here so we observed suppressed overpotentials by hydrogen bond interactions in all of them (Figures S24-S30). Therefore, introducing N-rich sites to COFs and establishing hydrogen bond interactions in $\mathrm{H}^{*}$ could be a promising strategy to achieve metal-free hydrogen evolution, and this method applies to 
other organic photocatalysts as well, including linear polymers and CMPs.

In order to further validate that the photo-induced electron could drive the reduction of water in neutral solution $(\mathrm{pH}=7)$, we calculated the Gibbs free energy change on the N2 site in the presence and absence of light-induced bias potential $(U)$ respectively. As shown in Figure 4c, in the dark this bias potential is equal to zero and the overall reaction is up-hill in energy therefore forbidden thermodynamically. However, under the visible light irradiation the bias potential is about $0.8 \mathrm{eV}$ and the overall reaction turns downhill, which implies the N2 site of PPy-BT capable of metal-free catalysis for HER in neutral solution. We also noticed that adsorption of a $\mathrm{H}$ atom on the $\mathrm{N} 2$ site could reduce the overpotential of nearby sites on the same edge unit such as $\mathrm{C}, \mathrm{S}$, and $\mathrm{N} 1$ sites, and even activated the nearby $\mathrm{N} 1$ site under visible light which would accelerate the hydrogen production rate immensely (Figure 4d and Figure S31). This phenomenon can be explained by the formation of stable closed-shell intermediate after two hydrogen atoms are simultaneously adsorbed on COFs, which is consistent with the previous observation of lower overpotential at high hydrogen coverage. ${ }^{51}$ Altogether five D-A COFs out of eight showed promising metal-free photocatalytic activity for HER in neutral solution under visible light and they are PPy-BT, PPy-BT(F), PPy-PT, PPy-TzBI, and $P P y-Q$, while $P P y-Q(F)$ requires the load of Pt co-catalyst because of the high overpotential in it.

\section{CONCLUSIONS}

To conclude, we proposed a facile strategy to attain efficient and metal-free hydrogen production driven by visible light by tailoring 2D conjugated D-A COFs in Lieb lattice. Based on this strategy, we designed eight D-A COFs with pyrene donor as node and different acceptors as edge linked by $\mathrm{C}=\mathrm{C}$. First of all, we demonstrated the successful modulation of band gaps and band edge positions of D-A COFs towards visible light absorption and overall water splitting by regulating the acceptor edge units with deep frontier orbitals. Moreover, we found that D-A design led to space-separated electron-hole pairs and it increased the dielectric screening as compared to the D-D COF, thereby giving rise to the reduction of exciton binding energies. More importantly, we revealed the vital role of hydrogen bond interactions played in stabilizing the structure of $\mathrm{H}^{*}$ intermediate and suppressing the overpotential for HER, which provides a new scenario for the rational design of active sites on COFs for metal-free water splitting. We obtained five D-A COFs capable of visible-light-driven hydrogen generation in neutral solution and metal-free photocatalysis, which could be synthesized in lab via Knoevenagel polycondensation reaction and are promising candidates for future laboratory research. These findings not only shed light on how to design new COFs for efficient visiblelight-driven water splitting, but also pave the way to develop metalfree photocatalysts for other chemical transformations.

\section{METHODS}

We performed density functional theory (DFT) calculations to obtain all the electronic structures of COFs in this work, including optimization of both crystal structure parameters and atomic coordinates, as implemented in the Vienna ab-initio simulation package (VASP 5.3.5) ${ }^{54}$ using the projector augmented wave (PAW) ${ }^{55}$ method and Perdew-Burke-Ernzerhof (PBE) ${ }^{56}$ exchange-correlation functional. Grimme's D3 approach was applied to include the London dispersion correction, ${ }^{57}$ and Heyd-Scuseria-Ernzerhof (HSE06) ${ }^{58}$ hybrid functional was used to obtain accurate band gaps. Further, GW method at the level of $\mathrm{G}_{0} \mathrm{~W}_{0}$ was employed to calcu- late the quasiparticle band gap and it was combined with Bethe-Salpeter equation (BSE) to calculate the optical band gap and exciton binding energy. In the lattice optimizations, a cut-off energy of $400 \mathrm{eV}$ for plane-wave basis set was employed while in the static calculations a cut-off energy of $600 \mathrm{eV}$ was used. And the convergence criterion of forces during atomic optimizations was $0.02 \mathrm{eV}^{-1}$. The energy convergence criterion in the self-consistent field iteration was $10^{-5} \mathrm{eV}$ in optimizations while $10^{-6} \mathrm{eV}$ in static calculations. The k-mesh of $1 \times 1 \times 5$ was used in the structural relaxation of COFs while denser k-mesh of $1 \times 1 \times 8$ was employed to calculate the converged charge density. To model the hydrogen evolution reaction, a $1 \times 1 \times 3$ supercell of COFs was constructed, and hydrogen atoms were adsorbed to different sites on the edge unit. A k-mesh of $1 \times 1 \times 3$ was used to optimize the structure. Computational hydrogen electrode model was applied and Gibbs free energies were obtained using the approach proposed by Nørskov. ${ }^{51}$ The spin-polarization effect was considered in the calculation of hydrogen-adsorbed intermediate $\mathrm{H}^{*}$. The long-range corrected functional $\omega$ B97XD and the def2tzvp basis set were used to obtain the frontier orbital energies of building blocks of COFs by the Gaussian16 program package. ${ }^{59}$ 


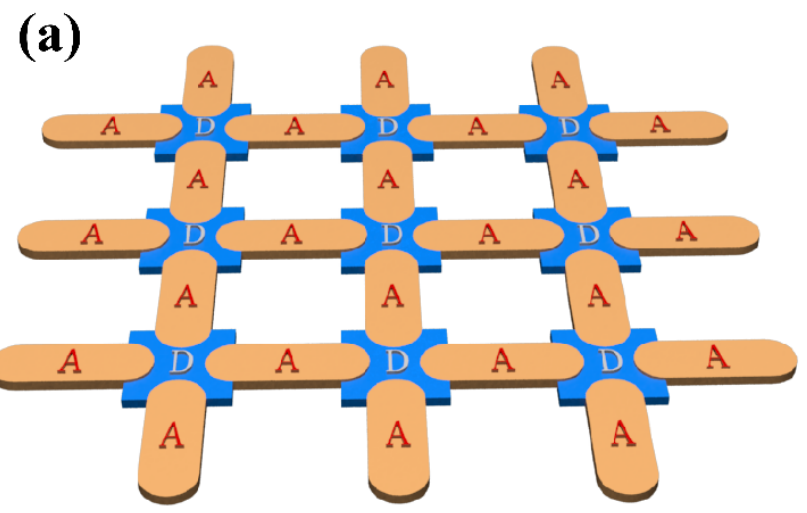

(a)

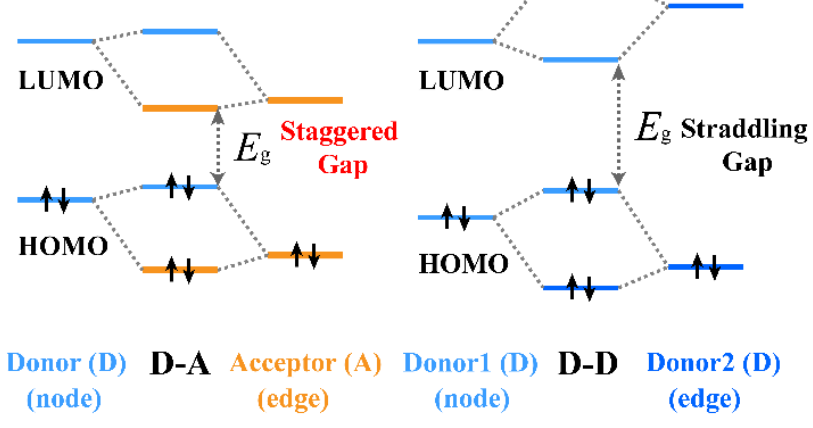

(b)

(b)

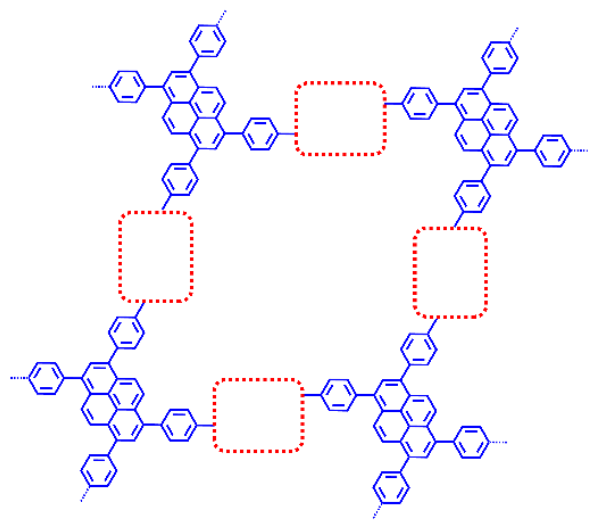

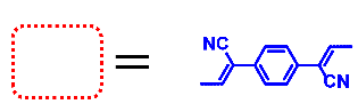
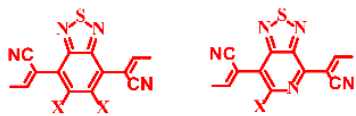

$\mathbf{P h}$

BT

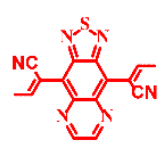

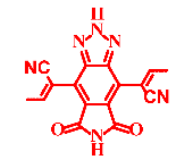

TzBI
PT

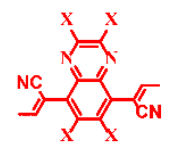

Q

Scheme 1. (a) Topological structure and building blocks of 2D COFs with donor nodes (blue) and acceptor edges (orange). (b) Chemical structures of PPy-Ph, PPy-BT, PPy-PT, PPy-TDQ, PPy-TzBI and PPy-Q. PPy represents the pyrene node and it is short for tetrakis(4-formylphenyl)phenyl. Ph, BT, PT, TDQ, TzBI, and $Q$ represent the edges, which are short for phenyl, benzothiadiazole, pyridal[2,1,3]thiadiazole, thiadiazoloquinoxaline, pyrrolo[3,4-f]benzotriazole-5,7-dione, and quinoxaline, respectively. $\mathrm{X}=-\mathrm{H}$ or $-\mathrm{F}$.

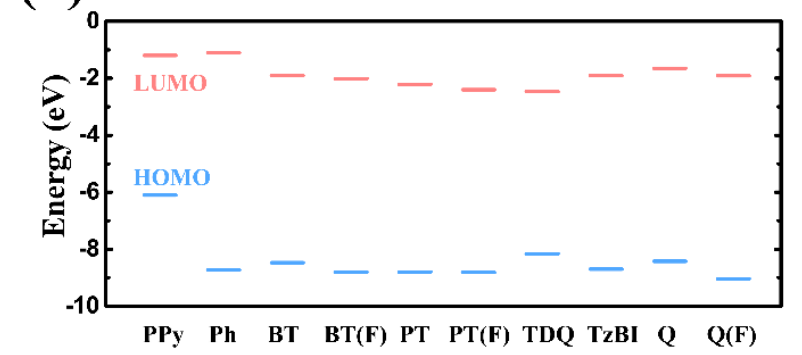

(c)

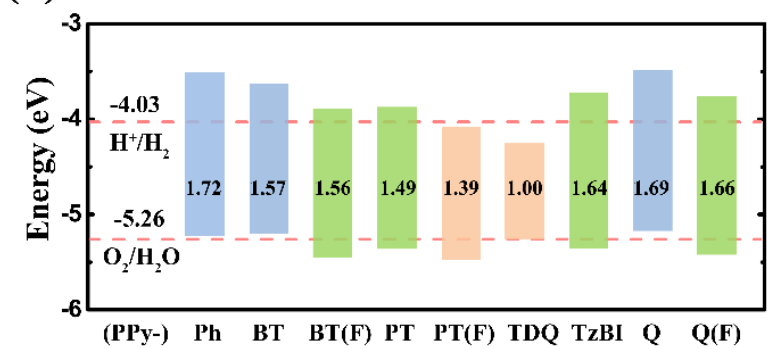

(d)

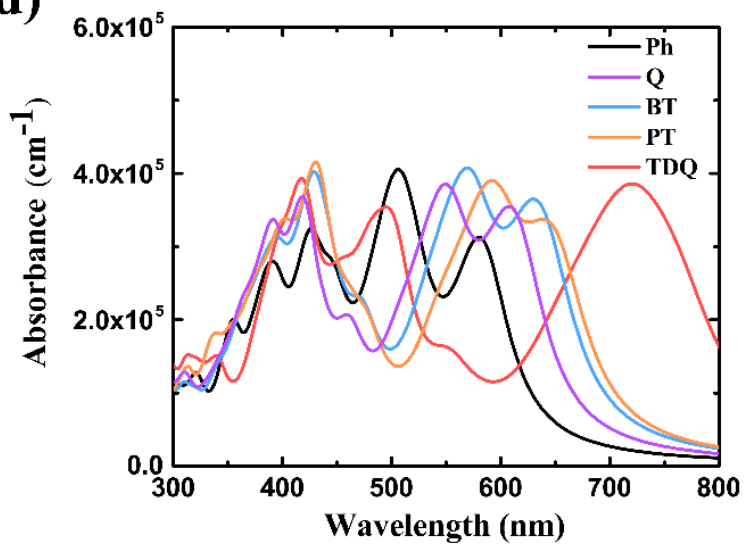

Figure 1. (a) Two types of band gap in COFs and corresponding energy level alignment of frontier orbitals in building blocks. (b) HOMO and LUMO energy level diagram for building blocks of COFs. PPy represents the pyrene node, and Ph, BT, BT(F), PT, PT(F), TDQ, TzBI, Q, and $Q(F)$ represent edges of COFs. (c) Energies of VBM and CBM in COFs relative to the vacuum level calculated with the HSE06 functional. The dashed lines represent redox potentials of water at $\mathbf{p H}=7$. Band gaps are also shown in the figure. COFs capable of HER, OER and overall water splitting are shown in blue, orange and 
green, respectively. (d) Optical absorption spectra of PPy-Ph, PPy-BT, PPy-PT, PPy-TDQ, and PPy-Q calculated with the $\mathrm{G}_{0} \mathrm{~W}_{0}+\mathrm{BSE}$ method.

(a)

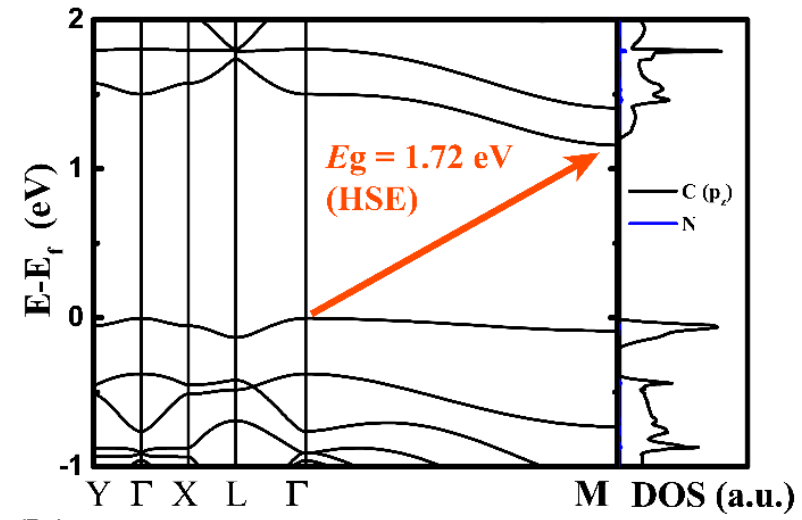

(b)

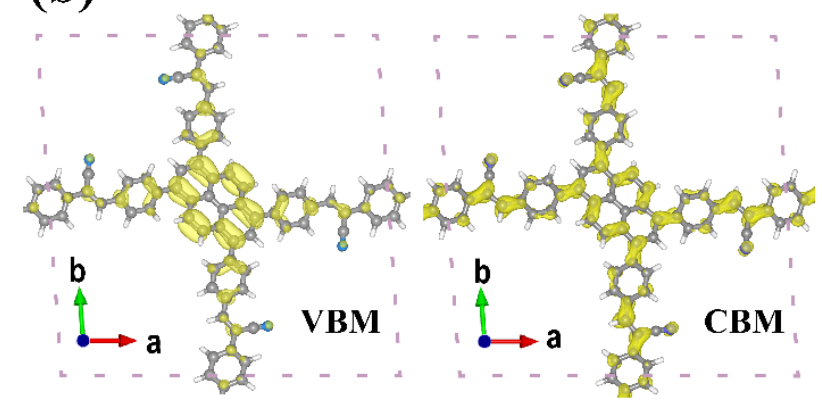

(c)

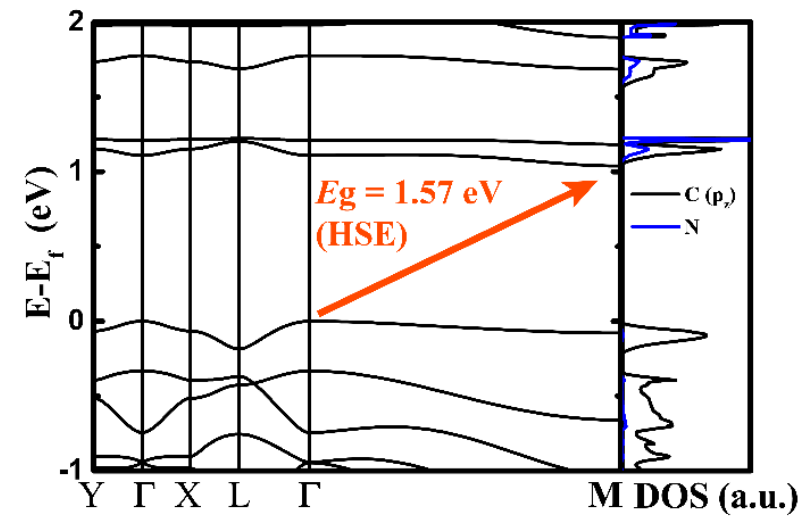

(d)

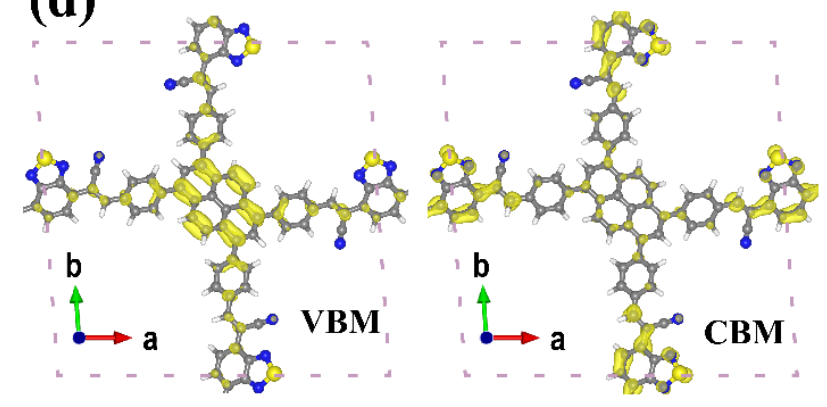

Figure 2. (a) Band structure and (b) partial density of states of PPh-Ph, a D-D COF. (c) Band structure and (d) partial density of states of PPy-BT, a D-A COF. The coordinates of high-symmetry points are $Y:(0.5,0,0), \Gamma:(0,0,0), X:(0,0.5,0), L:(0.5$, $0.5,0), M:(0,0,0.5)$. Charge density at VBM is shown on the left panel and $C B M$ on the right. Band structure was calculated with the PBE-D3 functional, and band gap calculated with the HSE functional was provided in the figure. 
(a)

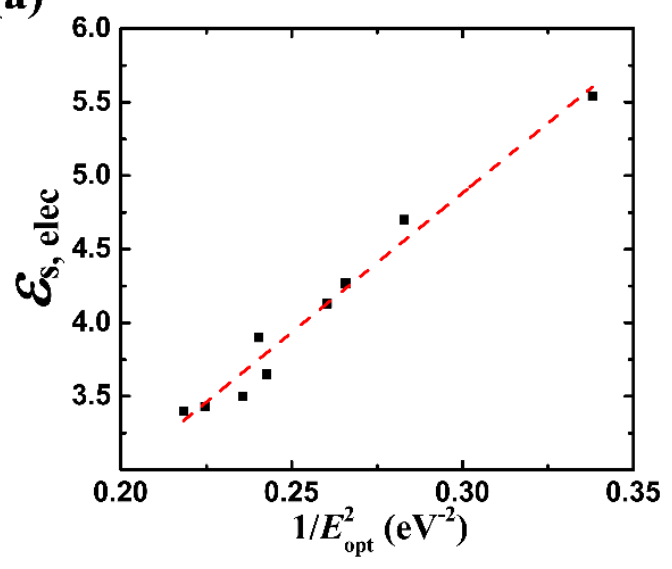

(c)

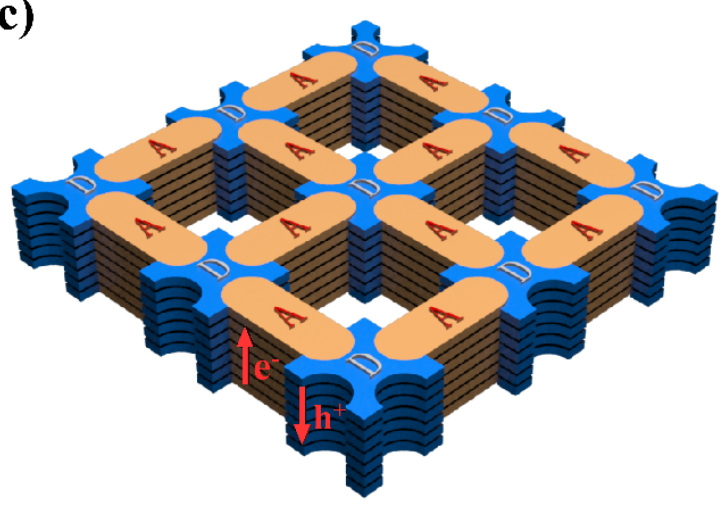

(b)

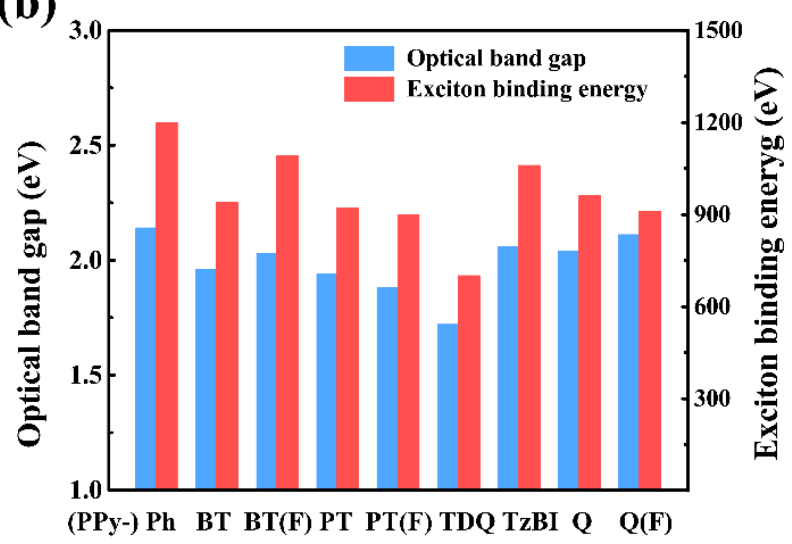

(d)

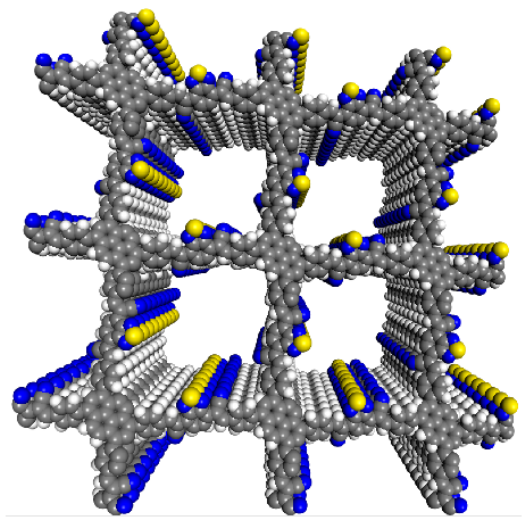

Figure 3. (a) Relationship between the electronic static dielectric constant $\left(\varepsilon_{\mathrm{s} \text {, elec }}\right)$ and the optical band gap $\left(E_{\mathrm{opt}}=\hbar \omega_{\mathrm{e}}\right)$ of $\mathrm{COFs}$, well characterized by the formula $\varepsilon$ s, elec $=1+N \mathrm{e}^{2} /\left(m \varepsilon_{0} \omega_{\mathrm{e}}{ }^{2}\right)$. The red dashed line represents a linear fit of the data. (b) Positive correlation between the optical band gap and the exciton binding energy of COFs. (c) Schematic illustration of the stacking structure of D-A COFs and the separation of one-dimensional transport channels for electron and hole. (f) Stacking structure of PPy-BT. 
(a)
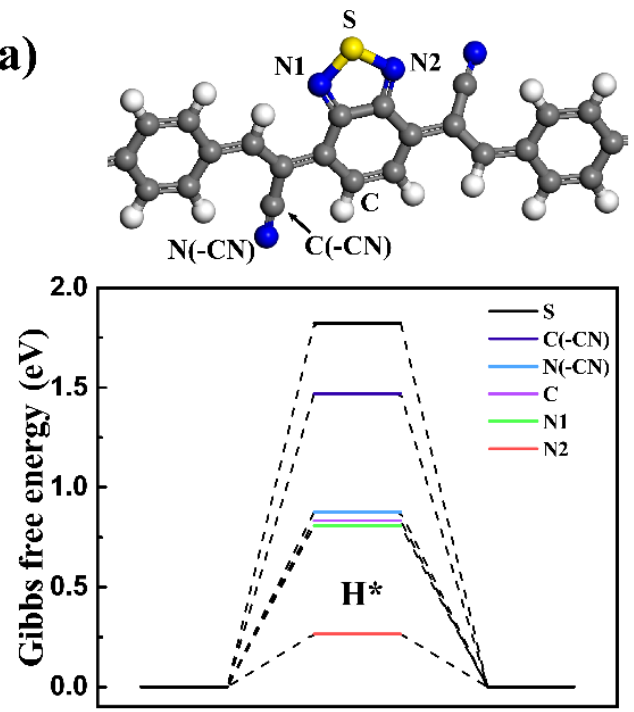

Reaction Coordinate

(c)

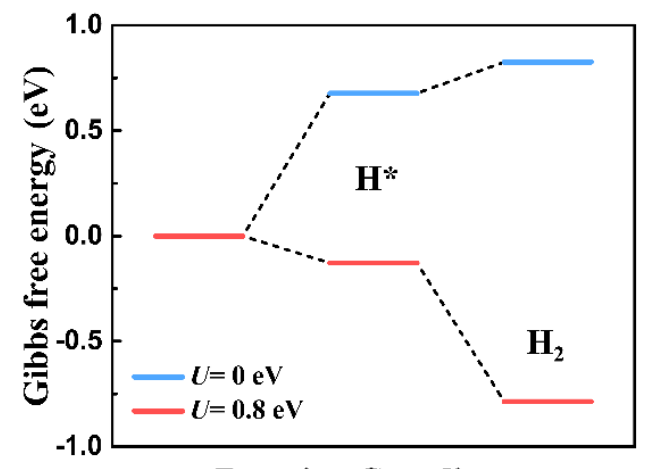

Reaction Coordinate (b)

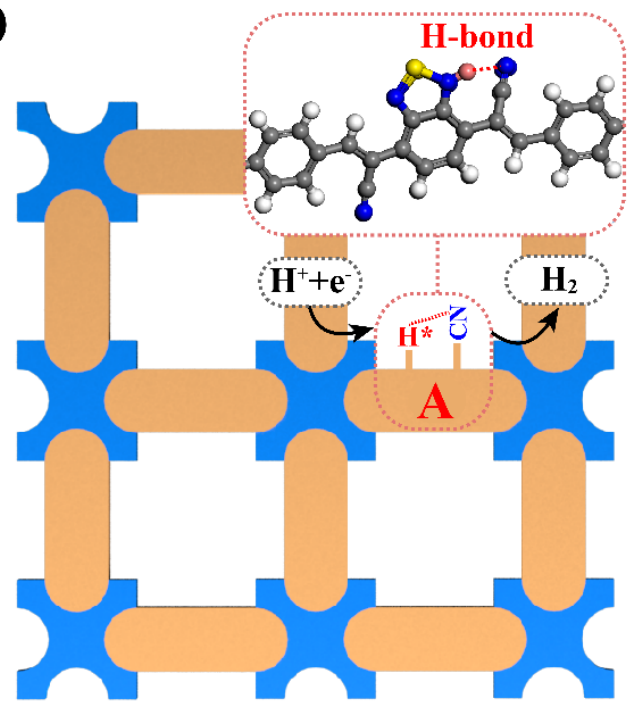

(d)

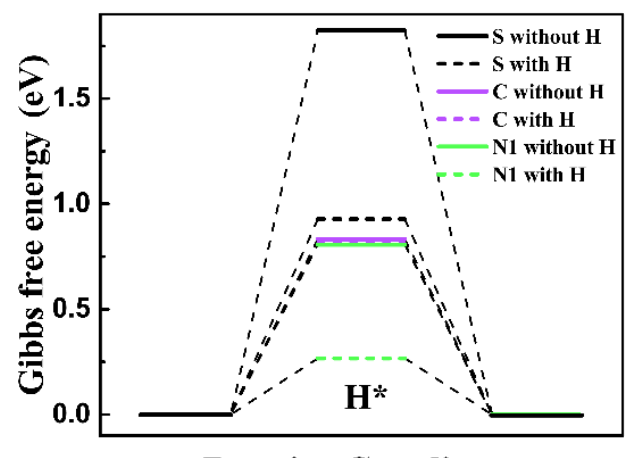

Figure 4. (a) Gibbs free energy changes of metal-free HER process on different catalytic sites of PPy-BT at pH = 0. (b) Structural illustration of intermediate $\left(\mathrm{H}^{*}\right)$ with hydrogen adsorption on $\mathrm{N} 2$ site of PPy-BT and formation of a hydrogen bond with the nearby $\mathrm{N}(-\mathrm{CN})$. (c) Gibbs free energy changes of HER process on $\mathrm{N} 2$ site of PPy-BT at $\mathrm{pH}=7$ with $U=0$ and $0.8 \mathrm{eV}$ respectively. (d) Gibbs free energy changes on different catalytic sites with or without hydrogen atom on the N2 site. 
Table 1. Electronic and optical properties of PPy-Ph and designed D-A COFs

\begin{tabular}{|c|c|c|c|c|c|c|c|c|}
\hline (PPy-) & $E_{\mathrm{g}}(\mathrm{eV})$ & $E_{\text {opt }}(\mathrm{eV})$ & VBM $(\mathrm{eV})$ & CBM $(\mathrm{eV})$ & $m_{\mathrm{h}}{ }^{*} / m_{\mathrm{e}}$ & $m_{\mathrm{e}}^{*} / m_{\mathrm{e}}$ & $\varepsilon_{\mathrm{s}}$ & $E_{\mathrm{b}}(\mathrm{meV})$ \\
\hline $\mathrm{Ph}$ & 1.72 & 2.14 & -5.23 & -3.51 & 0.54 & 0.34 & 3.60 & 1200 \\
\hline $\mathrm{BT}$ & 1.57 & 1.96 & -5.20 & -3.63 & 0.40 & 0.38 & 4.50 & 940 \\
\hline $\mathrm{BT}(\mathrm{F})$ & 1.56 & 2.03 & -5.45 & -3.89 & 0.60 & 0.44 & 4.00 & 1090 \\
\hline PT & 1.49 & 1.94 & -5.36 & -3.87 & 0.43 & 0.76 & 4.63 & 920 \\
\hline PT(F) & 1.39 & 1.88 & -5.48 & -4.09 & 0.39 & 0.71 & 5.12 & 900 \\
\hline TDQ & 1.00 & 1.72 & -5.26 & -4.26 & 0.39 & 1.09 & 6.05 & 700 \\
\hline TzBI & 1.64 & 2.06 & -5.36 & -3.72 & 0.96 & 0.53 & 4.02 & 1060 \\
\hline Q & 1.69 & 2.04 & -5.18 & -3.48 & 0.44 & 0.62 & 4.22 & 960 \\
\hline $\mathrm{Q}(\mathrm{F})$ & 1.66 & 2.11 & -5.42 & -3.76 & 0.80 & 0.46 & 4.65 & 910 \\
\hline
\end{tabular}

\section{ASSOCIATED CONTENT}

\section{Supporting Information}

The Supporting Information is available free of charge on the ACS Publication website http://pubs.acs.org. Band structure, pDOS, charge densities, and absorption spectra of of PPy-BT(F), PPyPT, PPy-PT(F), PPy-TDQ, PPy-TzBI, PPy-Q, and PPy-Q(F), Gibbs free energy changes for HER

\section{AUTHOR INFORMATION}

\section{Corresponding Author}

*E-mail: (D. W.) dong913@mail.tsinghua.edu.cn

ORCID

Dong Wang: 0000-0002-0594-0515

Hongde Yu: 0000-0002-2576-271X

Notes

The authors declare no competing financial interest.

\section{ACKNOWLEDGMENT}

This work is supported by the National Natural Science Foundation of China (Grant Nos. 22073055 and 21673123). Computational resources are provided by the Tsinghua Supercomputing Center.

\section{REFERENCES}

(1) Hisatomi, T.; Domen, K. Reaction Systems for Solar Hydrogen Production Via Water Splitting with Particulate Semiconductor Photocatalysts. Nat. Catal. 2019, 2, 387-399.

(2) Kudo, A.; Miseki, Y. Heterogeneous Photocatalyst Materials for Water Splitting. Chem. Soc. Rev. 2009, 38, 253-278.

(3) Wang, Y.; Suzuki, H.; Xie, J.; Tomita, O.; Martin, D. J.; Higashi, M.; Kong, D.; Abe, R.; Tang, J. Mimicking Natural Photosynthesis: Solar to Renewable $\mathrm{H}_{2}$ Fuel Synthesis by Z-Scheme Water Splitting Systems. Chem. Rev. 2018, 118, 5201-5241.

(4) Sivula, K.; van de Krol, R. Semiconducting Materials for Photoelectrochemical Energy Conversion. Nat. Rev. Mater. 2016, 1, 15010. (5) Fujishima, A.; Honda, K. Electrochemical Photolysis of Water at a Semiconductor Electrode. Nature 1972, 238, 37-38.
(6) Chen, S.; Takata, T.; Domen, K. Particulate Photocatalysts for Overall Water Splitting. Nat. Rev. Mater. 2017, 2, 17050.

(7) Wang, Q.; Hisatomi, T.; Jia, Q.; Tokudome, H.; Zhong, M.; Wang, C.; Pan, Z.; Takata, T.; Nakabayashi, M.; Shibata, N.; Li, Y.; Sharp, I. D.; Kudo, A.; Yamada, T.; Domen, K. Scalable Water Splitting on Particulate Photocatalyst Sheets with a Solar-to-Hydrogen Energy Conversion Efficiency Exceeding 1\%. Nat. Mater. 2016, 15, 611-615.

(8) Wang, X.; Maeda, K.; Thomas, A.; Takanabe, K.; Xin, G.; Carlsson, J. M.; Domen, K.; Antonietti, M. A Metal-Free Polymeric Photocatalyst for Hydrogen Production from Water under Visible Light. Nat. Mater. 2009, 8, 76-80.

(9) Sprick, R. S.; Jiang, J.-X.; Bonillo, B.; Ren, S.; Ratvijitvech, T.; Guiglion, P.; Zwijnenburg, M. A.; Adams, D. J.; Cooper, A. I. Tunable Organic Photocatalysts for Visible-Light-Driven Hydrogen Evolution. $J$. Am. Chem. Soc. 2015, 137, 3265-3270.

(10) Woods, D. J.; Sprick, R. S.; Smith, C. L.; Cowan, A. J.; Cooper, A. I. A Solution-Processable Polymer Photocatalyst for Hydrogen Evolution from Water. Adv. Energy Mater. 2017, 7, 1700479.

(11) Sprick, R. S.; Bonillo, B.; Clowes, R.; Guiglion, P.; Brownbill, N. J.; Slater, B. J.; Blanc, F.; Zwijnenburg, M. A.; Adams, D. J.; Cooper, A. I. Visible-Light-Driven Hydrogen Evolution Using Planarized Conjugated Polymer Photocatalysts. Angew. Chem. Int. Ed. 2016, 128, 1824-1828.

(12) Zhang, G.; Lan, Z. A.; Wang, X. Conjugated Polymers: Catalysts for Photocatalytic Hydrogen Evolution. Angew. Chem. Int. Ed. 2016, 55, 15712-15727.

(13) Thote, J.; Aiyappa, H. B.; Deshpande, A.; Diaz Diaz, D.; Kurungot, S.; Banerjee, R. A Covalent Organic Framework-Cadmium Sulfide Hybrid as a Prototype Photocatalyst for Visible-Light-Driven Hydrogen Production. Chem. Eur. J. 2014, 20, 15961-15965.

(14) Vyas, V. S.; Haase, F.; Stegbauer, L.; Savasci, G.; Podjaski, F.; Ochsenfeld, C.; Lotsch, B. V. A Tunable Azine Covalent Organic Framework Platform for Visible Light-Induced Hydrogen Generation. Nat. Commun. 2015, 6, 8508.

(15) Sick, T.; Hufnagel, A. G.; Kampmann, J.; Kondofersky, I.; Calik, M.; Rotter, J. M.; Evans, A.; Döblinger, M.; Herbert, S.; Peters, K.; Böhm, D.; Knochel, P.; Medina, D. D.; Fattakhova-Rohlfing, D.; Bein, T. Oriented Films of Conjugated 2d Covalent Organic Frameworks as Photocathodes for Water Splitting. J. Am. Chem. Soc. 2017, 140, 2085-2092.

(16) Banerjee, T.; Gottschling, K.; Savasci, G. k.; Ochsenfeld, C.; Lotsch, B. V. $\mathrm{H}_{2}$ Evolution with Covalent Organic Framework Photocatalysts. ACS Energy Lett. 2018, 3, 400-409.

(17) Wang, X.; Chen, L.; Chong, S. Y.; Little, M. A.; Wu, Y.; Zhu, W.-H.; Clowes, R.; Yan, Y.; Zwijnenburg, M. A.; Sprick, R. S.; Cooper, A. I. Sulfone-Containing Covalent Organic Frameworks for Photocatalytic Hydrogen Evolution from Water. Nat. Chem. 2018, 10, 1180-1189 
(18) Jin, E.; Lan, Z.; Jiang, Q.; Geng, K.; Li, G.; Wang, X.; Jiang, D. 2d Sp² Carbon-Conjugated Covalent Organic Frameworks for Photocatalytic Hydrogen Production from Water. Chem 2019, 5, 1632-1647.

(19) Pachfule, P.; Acharjya, A.; Roeser, J.; Langenhahn, T.; Schwarze, M.; Schomäcker, R.; Thomas, A.; Schmidt, J. Diacetylene Functionalized Covalent Organic Framework (Cof) for Photocatalytic Hydrogen Generation. J. Am. Chem. Soc. 2018, 140, 1423-1427.

(20) Stegbauer, L.; Zech, S.; Savasci, G.; Banerjee, T.; Podjaski, F.; Schwinghammer, K.; Ochsenfeld, C.; Lotsch, B. V. Tailor-Made Photoconductive Pyrene-Based Covalent Organic Frameworks for VisibleLight Driven Hydrogen Generation. Adv. Energy Mater. 2018, 8, 1703278. (21) Waller, P. J.; Gándara, F.; Yaghi, O. M. Chemistry of Covalent Organic Frameworks. Acc. Chem. Res. 2015, 48, 3053-3063.

(22) Cote, A. P.; Benin, A. I.; Ockwig, N. W.; O'Keeffe, M.; Matzger, A. J.; Yaghi, O. M. Porous, Crystalline, Covalent Organic Frameworks. Science 2005, 310, 1166-1170.

(23) Li, X.; Yadav, P.; Loh, K. P. Function-Oriented Synthesis of TwoDimensional (2d) Covalent Organic Frameworks-from 3d Solids to 2d Sheets. Chem. Soc. Rev. 2020, 49, 4835-4866.

(24) Chen, X.; Geng, K.; Liu, R.; Tan, K. T.; Gong, Y.; Li, Z.; Tao, S.; Jiang, Q.; Jiang, D. Covalent Organic Frameworks: Chemical Approaches to Designer Structures and Built-in Functions. Angew. Chem. Int. Ed. 2020, 59, 5050-5091.

(25) Huang, N.; Wang, P.; Jiang, D. Covalent Organic Frameworks: A Materials Platform for Structural and Functional Designs. Nat. Rev. Mater. 2016, 1, 16068.

(26) Ghosh, S.; Nakada, A.; Springer, M. A.; Kawaguchi, T.; Suzuki, K.; Kaji, H.; Baburin, I.; Kuc, A.; Heine, T.; Suzuki, H.; Abe, R.; Seki, S. Identification of Prime Factors to Maximize the Photocatalytic Hydrogen Evolution of Covalent Organic Frameworks. J. Am. Chem. Soc. 2020, 9752 9762.

(27) Gottschling, K.; Savasci, G. k.; Vignolo-González, H.; Schmidt, S.; Mauker, P.; Banerjee, T.; Rovó, P.; Ochsenfeld, C.; Lotsch, B. V. Rational Design of Covalent Cobaloxime-Covalent Organic Framework Hybrids for Enhanced Photocatalytic Hydrogen Evolution. J. Am. Chem. Soc. 2020, 142, 12146-12156.

(28) Chen, W.; Wang, L.; Mo, D.; He, F.; Wen, Z.; Wu, X.; Xu, H.; Chen, L. Modulating Benzothiadiazole-Based Covalent Organic Frameworks Via Halogenation for Enhanced Photocatalytic Water Splitting. Angew. Chem. Int. Ed. 2020, 59, 16902-16909.

(29) Wei, S.; Zhang, F.; Zhang, W.; Qiang, P.; Yu, K.; Fu, X.; Wu, D.; Bi, S.; Zhang, F. Semiconducting 2d Triazine-Cored Covalent Organic Frameworks with Unsubstituted Olefin Linkages. J. Am. Chem. Soc. 2019, 141, 14272-14279.

(30) Wan, Y.; Wang, L.; Xu, H.; Wu, X.; Yang, J. A Simple Molecular Design Strategy for Two-Dimensional Covalent Organic Framework Capable of Visible-Light-Driven Water Splitting. J. Am. Chem. Soc. 2020, 142, 4508-4516.

(31) Wang, Y.; Vogel, A.; Sachs, M.; Sprick, R. S.; Wilbraham, L.; Moniz, S. J. A.; Godin, R.; Zwijnenburg, M. A.; Durrant, J. R.; Cooper, A. I.; Tang, J. Current Understanding and Challenges of Solar-Driven Hydrogen Generation Using Polymeric Photocatalysts. Nat. Energy 2019, 4, 746-760. (32) Xu, S.; Sun, H.; Addicoat, M.; Biswal, B. P.; He, F.; Park, S.; Paasch, S.; Zhang, T.; Sheng, W.; Brunner, E.; Hou, Y.; Richter, M.; Feng, X. Thiophene-Bridged Donor-Acceptor $\mathrm{Sp}^{2}$-Carbon-Linked 2d Conjugated Polymers as Photocathodes for Water Reduction. Adv. Mater. 2021, 33 , 2006274.

(33) Li, X.; Gao, Q.; Aneesh, J.; Xu, H.-S.; Chen, Z.; Tang, W.; Liu, C.; Shi, X.; Adarsh, K.; Lu, Y.; Loh, K. P. Molecular Engineering of Bandgaps in Covalent Organic Frameworks. Chem. Mater. 2018, 30, 5743-5749.

(34) Liu, J.; Liu, Y.; Liu, N.; Han, Y.; Zhang, X.; Huang, H.; Lifshitz, Y.; Lee, S.-T.; Zhong, J.; Kang, Z. Metal-Free Efficient Photocatalyst for Stable Visible Water Splitting Via a Two-Electron Pathway. Science 2015, 347, 970-974.

(35) Li, S.; Li, L.; Li, Y.; Dai, L.; Liu, C.; Liu, Y.; Li, J.; Lv, J.; Li, P.; Wang, B. Fully Conjugated Donor-Acceptor Covalent Organic Frameworks for Photocatalytic Oxidative Amine Coupling and Thioamide Cyclization. ACS Catal. 2020, 10, 8717-8726.

(36) Chen, R.; Shi, J. L.; Ma, Y.; Lin, G.; Lang, X.; Wang, C. Designed Synthesis of a 2d Porphyrin-Based $\mathrm{Sp}^{2}$ Carbon-Conjugated Covalent Organic Framework for Heterogeneous Photocatalysis. Angew. Chem. Int. Ed. 2019, 58, 6430-6434.
(37) Fu, C.-F.; Wu, X.; Yang, J. Material Design for Photocatalytic Water Splitting from a Theoretical Perspective. Adv. Mater. 2018, 30, 1802106. (38) Vezie, M. S.; Few, S.; Meager, I.; Pieridou, G.; Dörling, B.; Ashraf, R. S.; Goñi, A. R.; Bronstein, H.; McCulloch, I.; Hayes, S. C.; Campoy-Quiles, M.; Nelson, J. Exploring the Origin of High Optical Absorption in Conjugated Polymers. Nat. Mater. 2016, 15, 746-753.

(39) Chen, L.; Furukawa, K.; Gao, J.; Nagai, A.; Nakamura, T.; Dong, Y.; Jiang, D. Photoelectric Covalent Organic Frameworks: Converting Open Lattices into Ordered Donor-Acceptor Heterojunctions. J. Am. Chem. Soc. 2014, 136, 9806-9809.

(40) Qian, Y.; Li, D.; Han, Y.; Jiang, H.-L. Photocatalytic Molecular Oxygen Activation by Regulating Excitonic Effects in Covalent Organic Frameworks. J. Am. Chem. Soc. 2020, 142, 20763-20771.

(41) Wang, H.; Jin, S.; Zhang, X.; Xie, Y. Excitonic Effects in Polymeric Photocatalysts. Angew. Chem. Int. Ed. 2020, 59, 22828-22839.

(42) Wang, H.; Liu, W.; Zhang, P.; He, X.; Zhang, X.; Xie, Y. Toward an Excitonic Perspective on Low-Dimensional Semiconductors for Photocatalysis. J. Am. Chem. Soc. 2020, 142, 14007-14022.

(43) Thomas, S.; Li, H.; Zhong, C.; Matsumoto, M.; Dichtel, W. R.; Bredas, J.-L. Electronic Structure of Two-Dimensional П-Conjugated Covalent Organic Frameworks. Chem. Mater. 2019, 31, 3051-3065.

(44) Springer, M. A.; Liu, T.-J.; Kuc, A.; Heine, T. Topological TwoDimensional Polymers. Chem. Soc. Rev. 2020, 49, 2007-2019.

(45) Nagai, A.; Guo, Z.; Feng, X.; Jin, S.; Chen, X.; Ding, X.; Jiang, D. Pore Surface Engineering in Covalent Organic Frameworks. Nat. Commun. 2011, 2, 1-8.

(46) Martin, D. J.; Reardon, P. J. T.; Moniz, S. J.; Tang, J. Visible LightDriven Pure Water Splitting by a Nature-Inspired Organic SemiconductorBased System. J. Am. Chem. Soc. 2014, 136, 12568-12571.

(47) Pandey, L.; Risko, C.; Norton, J. E.; Bredas, J.-L. Donor-Acceptor Copolymers of Relevance for Organic Photovoltaics: A Theoretical Investigation of the Impact of Chemical Structure Modifications on the Electronic and Optical Properties. Macromolecules 2012, 45, 6405-6414.

(48) Bredas, J.-L. Mind the Gap! Mater. Horiz. 2014, 1, 17-19.

(49) Cho, N.; Schlenker, C. W.; Knesting, K. M.; Koelsch, P.; Yip, H. L.; Ginger, D. S.; Jen, A. K. Y. High-Dielectric Constant Side-Chain Polymers Show Reduced Non-Geminate Recombination in Heterojunction Solar Cells. Adv. Energy Mater. 2014, 4, 1301857.

(50) Cox, P. A., The Electronic Structure and Chemistry of Solids. Oxford University Press: 1987.

(51) Nørskov, J. K.; Bligaard, T.; Logadottir, A.; Kitchin, J.; Chen, J. G.; Pandelov, S.; Stimming, U. Trends in the Exchange Current for Hydrogen Evolution. J. Electrochem. Soc. 2005, 152, J23.

(52) Jin, E.; Asada, M.; Xu, Q.; Dalapati, S.; Addicoat, M. A.; Brady, M. A.; Xu, H.; Nakamura, T.; Heine, T.; Chen, Q.; Jiang, D. Two-Dimensional $\mathrm{Sp}^{2}$ Carbon-Conjugated Covalent Organic Frameworks. Science 2017, 357, 673-676.

(53) Zhuang, X.; Zhao, W.; Zhang, F.; Cao, Y.; Liu, F.; Bi, S.; Feng, X. A Two-Dimensional Conjugated Polymer Framework with Fully Sp²-Bonded Carbon Skeleton. Polym. Chem. 2016, 7, 4176-4181.

(54) Kresse, G.; Furthmüller, J. Efficient Iterative Schemes for Ab Initio Total-Energy Calculations Using a Plane-Wave Basis Set. Phys. Rev. B 1996, 54, 11169

(55) Blöchl, P. E. Projector Augmented-Wave Method. Phys. Rev. B 1994, 50,17953

(56) Perdew, J. P.; Burke, K.; Ernzerhof, M. Generalized Gradient Approximation Made Simple. Phys. Rev. Lett. 1996, 77, 3865.

(57) Grimme, S.; Antony, J.; Ehrlich, S.; Krieg, H. A Consistent and Accurate Ab Initio Parametrization of Density Functional Dispersion Correction (Dft-D) for the 94 Elements H-Pu. J. Chem. Phys. 2010, 132, 154104.

(58) Heyd, J.; Scuseria, G. E.; Ernzerhof, M. Hybrid Functionals Based on a Screened Coulomb Potential. J. Chem. Phys. 2003, 118, 8207-8215.

(59) Frisch, M. J.; Trucks, G. W.; Schlegel, H. B.; Scuseria, G. E.; Robb, M. A.; Cheeseman, J. R.; Scalmani, G.; Barone, V.; Petersson, G. A.; Nakatsuji, H.; Li, X.; Caricato, M.; Marenich, A. V.; Bloino, J.; Janesko, B. G.; Gomperts, R.; Mennucci, B.; Hratchian, H. P.; Ortiz, J. V.; Izmaylov, A. F.; Sonnenberg, J. L.; Williams; Ding, F.; Lipparini, F.; Egidi, F.; Goings, J.; Peng, B.; Petrone, A.; Henderson, T.; Ranasinghe, D.; Zakrzewski, V. G.; Gao, J.; Rega, N.; Zheng, G.; Liang, W.; Hada, M.; Ehara, M.; Toyota, K.; Fukuda, R.; Hasegawa, J.; Ishida, M.; Nakajima, T.; Honda, Y.; Kitao, O.; Nakai, H.; Vreven, T.; Throssell, K.; Montgomery Jr., J. A.; Peralta, J. E.; Ogliaro, F.; Bearpark, M. J.; Heyd, J. J.; Brothers, E. N.; Kudin, K. N.; 
Staroverov, V. N.; Keith, T. A.; Kobayashi, R.; Normand, J.; Raghavachari, K.; Rendell, A. P.; Burant, J. C.; Iyengar, S. S.; Tomasi, J.; Cossi, M.; Millam, J. M.; Klene, M.; Adamo, C.; Cammi, R.; Ochterski, J. W.; Martin,
R. L.; Morokuma, K.; Farkas, O.; Foresman, J. B.; Fox, D. J. Gaussian 16 Rev. C.01, Wallingford, CT, 2016. 


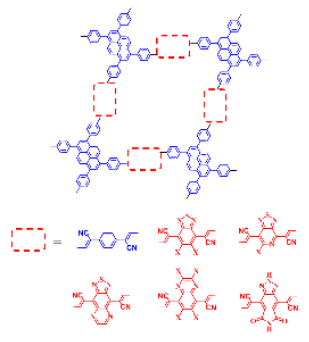

D-A COFs

in

Lieb lattice

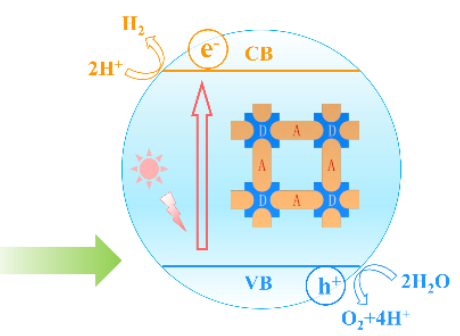

Enhanced sunlight absorption

Prompt exciton dissociation

Metal-free catalysis 